\title{
LA-UR- $\overrightarrow{9} 5-280 \%$
}

ImE $\quad$ SCALE UP OF FLOW IN POROUS MEDIA

AURHOR(S): Lianjun An, University at Stony Brook

James Glimm, University at Stony Brook

David H. Sharp, T-13

Qiang Zhang, University at Stony Brook

SUвMrrIEto: Proceedings of the 7th International Conference on Fluid Flow in

Porous Media, May 1995, St. Etienne, France
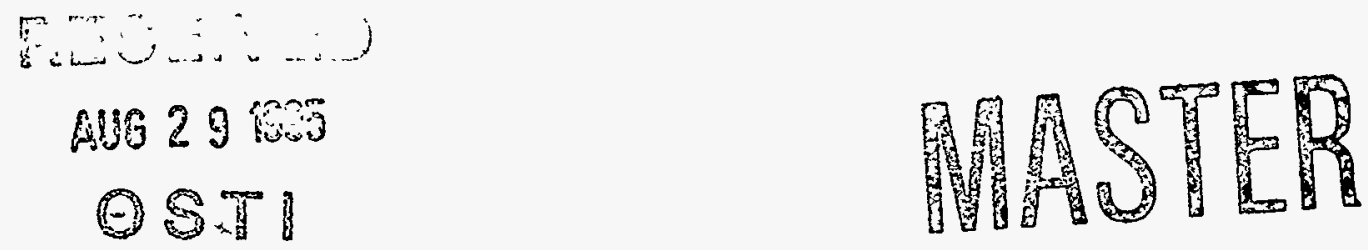

By acceptance of this article, the pubisher recognizes that the U.S. Goverment retains a nonexclusive, royalty-fiee license to publish or reproduce the published form of this contibution, or to allow others to do so, for U.S. Government purposes.

The Los Alamos National Laboratory requests that the pubisher identify this artide as work performed under the auspices of the U.S. Department of Energy.

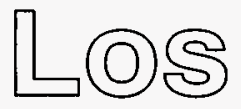

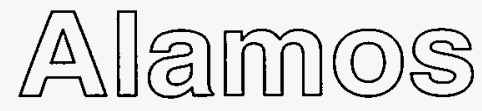

FORM NO. 836 R4

ST. NO. $26295 / 81$

\section{Los Alamos National Laboratory Los Alamos, New Mexico 87545}

DISTRIBUTION OF THIS DOCUMENT IS UNLIMITED 


\section{DISCLAIMER}

This report was prepared as an account of work sponsored by an agency of the United States Government. Neither the United States Government nor any agency thereof, nor any of their employees, makes any warranty, express or implied, or assumes any legal liability or responsibility for the accuracy, completeness, or usefuiness of any information, apparatus, product, or process disclosed, or represents that its use would not infringe privately owned rights. Reference herein to any specific commercial product, process, or service by trade name, trademark, manufacturer, or otherwise does not necessarily constitute or imply its endorsement, recommendation, or favoring by the United States Government or any agency thereof. The views and opinions of authors expressed herein do not necessarily state or reflect those of the United States Government or any agency thereof. 


\section{DISCLAIMER}

Portions of this document may be illegible in electronic image products. Images are produced from the best available original document. 


\title{
SCALE LP OF FLOWI IN POROLS MEDIA
}

\author{
Lianjun An. ${ }^{2}$ James Glimm, ${ }^{2} 3$ David H. Sharp. ${ }^{4}$ Qiang Zhang ${ }^{2}$
}

\begin{abstract}
We study the scale up problem for flow in porous media. The general nature of this problem is outlined, leading to a discussion of assumptions on random fields appropriate for the description of geological heterogeneities.

The main point of this paper is to use direct numerical simulation to evaluate the ensemble averages describing fluid dispersion. for flow in porous media. The relation between ensemble dispersion and single realization dispersion is discussed in the case of linear transport. and the role of plume or channel width is also explored. Finally: we consider nonlinear transport, and contrast dispersive to hyperbolic renormalization of the flow equations. For the geological and fluid paramters considered here. the hyperbolic renormalization is trivial, indicating that dispersive renormalization is appropriate in these cases.

Further study of the ideas explored in this paper will be required for a proper understanding of their role in a more complete theory which we hope will follow.
\end{abstract}

Key words: Porous Media. Multiphase Flow, Scale tip.

\section{Introduction}

The scale up problem for fl : $:$ in porous media addresses a central weakness in simulation models. In these models. there is a failure of one of the basic paradigms of modern technology, in which physical constants are measured in the laboratory, and then inserted into simulation models based on partial differential equations to predict field scale phenomena. The failure is due to the presence of intermediate variability or stochasticity, between the laboratory and field scales, and which is needed for valid field scale predictions. Consider the following facts: (a) The media is variable on all length scales. (b) The short length scale variation is known at best in terms of a probability model. (c) The short scale variation of the media influences the macroscopic, field scale flow behavior. (d) The geostatistics of variability enters the flow equations nonlinearly, as equation coefficients. and moreover. the flow equations are themselves usually nonlinear. Together these facts imply that ensemble averaged conditional simulation is needed. each realization of which contains fine grid heterogeneity information. Consequently, an increase in simulation speed by ord. : of magnitude is needed. Parallel computing is one response to this requirement. Here ; smphasize improved scientific understanding and simulation algorithms. as another response. The thrust

\footnotetext{
${ }^{1}$ Supported by DOE office of Scientific Computing through Martin Marietta Energy Systems Subcontract $28 \mathrm{XSK} 964 \mathrm{C}$

'The University at Stony Brook, Stony Brook, NY 11794-3600

${ }^{3}$ Also supported by the Applied Mathematics Subprogram of the U.S. Department of Energy DE-FG0290ER25084, the Army Research Office, grant DAAL03-92-G-0185 and through the Mathematical Sciences Institute of Cornell Lniversity under subcontract to the University at Stony Brook. ARO contract number DAAL03-91-C-0027, and the National Science Foundation. grant DMS-9201581.

${ }^{4}$ Complex Systems Group. Los Alamos National Laboratory, Los Alamos NM 87.54 .5
} 
of the required scientific understanding concerns the stochastic aspects of fuid flow in porous media.

The basic idea of scale up is to simulate on a coarser grid. with modified reservoir equations. In the procedure. the influence of fine scale variation, specified on a fine grid and with primitive reservoir equations, is captured in the coarse grid reservoir functions. The definition of pseudo. or effective permeabilities is one approact to the problem of constructing the modified coarse grid equations. It suffers from process and geology dependence. as well as cost, as a full fine grid simulation must be performed in this method. Homogenization theory addresses scale up in the idealized limit in which the fine scale variation is periodic and the fine and coarse scales are infinitely separated. In this limit, analytic formulas are possible. The requirement of fine scale periodicity can be regarded as a technical requirement. The validity of the theory is presumably independent of this restriction. Unfortunately, the same expectation does not apply to the requirement of infinite scale separation. The most important variation occurs on scales close to the observational length scales. and homogenization does not apply to these. Moment expansions, as in turbulence closure models. are another approach to scale up. Perturbation theory (in powers of the geology or velocity fluctuations) are another. Renormalized perturbation theory extends to some extent the domain of ralidity of primitive perturbation theory. The renormalization group provides another formalism for scale up. We will refer to some of these approaches, but uur main concern here will be the use of direct (fine scale) simulation to explore the solution space of the stochastic partial differential equations of porous media.

\section{Stochastic Geology and Fluid Initial Conditions}

The variety of geological mechanisms acting on various length scales support the idea that heterogeneity occurs on all length scales; observation of outcrops confirms this view. Geological variability is described in terms of a statistical model, using random fields. The current state of the art in geostatistical models is to use Boolean random fields (indicator functions) to specify the boundary between distinct rock types and stationary or stationary increment Gaussian random fields to specify variability within a single rock type. For simplicity here. we consider only Gaussian random fields. The most important geological variable is the permeability. $K$. Let $\xi=\ln K$ be the $\log$ permeability. Assuming that $\xi$ is Gaussian. and (after a suitable normalization) of mean zero, the statistical properties of $\xi$ are completely determined by its two point correlation function

$$
\langle\xi(x) \xi(y)\rangle=c(x, y) .
$$

For a stationary random field, $c=c(x-y)$ is a function of the two point separation $x-y$ alone, and the statistical properties of $\xi$ are translation invariant. A stationary increment random field is by definition one whose distributional derivative $\partial_{x} \xi$ is stationary. In the Gaussian case. a stationary increment random field is characterized by its variogram : $=$ $\gamma(x-y)=\left\langle(\xi(x)-\xi(y))^{2}\right\rangle$. Higher order stationary increment random fields are similarly defined, as random fields for which all higher derivatives of some specified order are stationary in their own right. 
As a simple model, let $\xi$ be isotropic. so that $c$ or $\hat{i}$ is a function of $r=\mid x-y_{i}$ alone: layering can be introduced by requiring $\xi$ to be isotropic in scaled variables $D x$. where the matrix $D$ is diagonal in the coordinates of the bedding plane $[\bar{\tau}, 10]$ To introduce variability over all length scales, we next restrict $\xi$ to be fractal, or self-similar. so that $c$ or $;$ is given by a power law. These fractal covariances are plausible representations of geolog: over intermediate length scales, as we explain below. They are attractive for two reasons: First, they at least qualitatively compatible with dispersivity data obtained from a large number of tracer floods [8] in which the dispersivity is observed to increase systematically with travel distance, perhaps in a power law relationship. Dispersivity is related to both the strength and the length scale of the heterogeneity encountered by the flood. With a larger travel distance, larger length scale heterogeneities are encountered, and contribute to the dispersivity. This phenomena has come to be known as "evolving heterogeneities". A second factor which makes the study of fractal covariances attractive is they are simple enough so that analytic information concerning the associated fluid flow can be obtained at least in some limiting cases. Since the numerical solution of stochastic partial differential equations. including convergence of ensemble averages as the number of realizations is increased, is a barely explored area of numerical analysis, even limited analytic information is extremely valuable as a check on the computational validity of the procedures employed. The stochastic partial differential equations of porous media fluid flow have spatially random coefficients. and thus are considerably more difficult to study than the stochasticity associated with a time dependent but spatially constant additive random term (Wiener measure).

Fractals have been proposed as a description of such diverse phenomena as flood stages on the Nile, tree rings and the geometry of clouds or coastlines. For a very limited range of situations, physics provides scaling laws valid over several decades. The critical point in statistical physics is an example. Such scaling laws are derived by a renormalization group equation analysis of fundamental physical equations, and they have striking universality properties. Scaling laws may also be regarded as a linear approximation to data which is smooth on log-log scales. This second point of view, while more general. carries fewer implications. In particular, the statement that a smooth curve has a tangent does not imply that the slope of the tangent is a universal number. To the extent that scaling laws model multi-length scale geostatistical heterogeneity, we believe it will be in the second sense. of a nonuniversal linear approximation of a smooth curve presented in $\log -\log$ coordinates.

A minimum mathematical and physical requirement on $K=\epsilon^{\xi}$ is that $K$ be positive. so that Darcy's law is well defined. According to the theory of distributions. this means that the random field $K^{\prime}$ must be at least a positive Borel measure in a.e. realization. To form the exponential, it would seem that the further requirement that $\xi$ be defined pointwise as a measurable function, presumably in $L_{1}$ loc , should be imposed. Lise of a Wick exponential [9] may allow relaxation of this condition, but the meaning of the implied subtractions should be examined before such constructions are adopted. In terms of allowed power laws. the $L_{1}{ }^{\text {loc }}$ requirement. or even the Borel measure requirement. is almost identical to the assumption that $\xi$ be Hölder continuous in a.e. realization. See for example [3. 4]. A sufficient requirement for the a.e. Hölder continuity property, for Gaussian random fields. is that the covariance $c(x, y)$ be Hölder continuous [11] In d space dimensions. $\xi$ will have a smaller Hölder exponent. by a factor smaller than $1 / 2 \mathrm{~d}$. and the example of Wiener measure 
shows that this bound cannot be improved upon. In the fractal case. we are then lead to the stationary increment processes. known in one dimension as fractional Brownian motion. Alternately: the short distance behavior should be regularized. and deviate from power law behavior. In practice. $K$ is generated numerically, and represented on a fine grid. The fine grid provides a short distance regularization.

However, fractal random fields which extrapolate to short distances smoothly have unphysical behavior at large distances. The stationary increment fields represent trends. They should be $\mathrm{u} \rightarrow \mathrm{d}$ for interpolation only, and not for long distance extrapolation. If the short distance belicuior is regular, then a Kriging restriction to condition the measure in terms of point values, e.g. at well locations, where a measurement has been taken, is defined as a measurable function of the ensemble. Similarly, history matching to pressure or production data at wells will constrain average or effective permeability values in regions surrounding the wells. Such measurements. if regularly spaced, will regularize the behavior of the conditioned ensemble at large distances. to the extent that the large distance behavior will resemble that of a stationary random field. We also allow the possibility of a long distance cutoff in the power law behavior. Alternately, we consider the concept of a multilength scale fractal. in which $c$ or $\gamma$ has the form $r^{-3}$. where $3=3(r)$ is a slowly varying function of $r$. For 3 varying sufficiently slowly. one can show [21] that scaling properties of the stochastic fluids flowing in a fractally random geology are equal to those of the frozen $3=$ con-t. geology over those length scales.

We note that if cutoffs are used to regularize fractal random fields. the cutoffs must be removed prior to evaluating any scaling laws which depend upon them. Otherwise. as demonstrated in [24.10] the scaling laws (e.g. those of [2] describing turbulent transport by a random velocity field) are nonunique, and reflect properties of the cutoffs in addition to those of the random velocity field power law. The cutoffs of [2] thus influence the scaling analysis. It is further explained [20] that the cutoffs [2] originate in a non-standard model of turbulent transport. in which the scaling, or inertial, range of turbulence has an explicit dependence upon the Reynolds number, i.e. the system viscosity. Virtually all theories of turbulence assume that the scaling properties of fully developed inertial range turbulence are independent of viscosity, and thus should be independent of cutoffs. as provided by viscosity and the finite Reynolds number.

The plume aspect ratio (length to width) influences dispersion scaling laws [18. 6] Carefully instrumented field studies ( $c f$. [19] of relatively homogeneous acquifers show transverse dispersivity smaller than longitudinal dispersivity by factors of three to ten. However. actual remediation sites are often geologically highly complex and heterogeneous and may be wider. The Hanford site. for example. shows a plume aspect ratio close to unity: the Columbus site [1] has a plume aspect ratio of about five.

Because transverse dispersivity affects the plume aspect ratio. it is a significant quantity: Theoretical determinations of scaling laws depend upon a perturbation expansion truncated at second order. The influence of transverse dispersivity upon longitudinal dispersivity does not enter at second order. so that consideration of higher order perturbation terms may be required to include the time dependent plume width effects. The use of renormalized expansions to include the effect of certa.: higher order terms and a more plausible treatment of larger amplitude perturbations, does. on some occasions. change the asymptotic scaling behavior from that based on primitive perturbation theory; truncated at second order. 


\section{The Dependence of Dispersion upon Flow Regimes}

\subsection{Linear Transport in Heterogeneous Media}

In a series of papers, the authors and coworkers have analyzed dispersivity for flow in porous media. These results. surveyed in [10] pertain to multi-length scale heterogeneities. such as fractals, and the emphasize a comparison between theory and computation. A typical result. shown in Figures 1 and 2, compares the computed mixing length to theoretical predictions based on ordinary perturbation theory. The methodology in this study is to start with an interface separating the fluid carrying the the passive tracer (tagged) from that which is not (untagged). A mean flow normal to the interface is assumed. The average concentration. as a function of time and longitudinal flow distance, is recorded, after transverse spatial averages and ensemble averages. This concentration, in its longitudinal spatial dependence, is fit to a complementary error function by least squares. The two parameters in this fit are those which characterize the complementary error function: mean position and mixing zone length. We believe that this methodology is essentially equivalent to the direct computation of the first and second moments of the fluid interface in the mixing zone. The geology was characterized by two parameters, an overall coefficient (coefficient of variation of the permeability). and an exponent (relative importance of short vs. long length scale heterogeneities). Two geology exponents and several geology coefficients were explored in this study.

The distinction between dispersion of the ensemble and the dispersion of a single realization is analyzed in $[5,18,6]$ to the level of second order primitive perturbation theory: See also the discussion of [10] To understand better the meaning of this distinction in the context of our methodology, we re-examine this question here. We note that the theory we have used is that of an infinitely wide plume. The computations were that of a plume extending to full channel width (no flow conditions at transverse boundaries), and in the case of slowly decaying geological heterogeneities ( $\beta=0.5$ in the covariance). with a width to maximum travel distance aspect ratio of $1: 1$. In Figure 3 we consider a computation in a narrower domain, with a channel width to maximal travel distance aspect ratio $1: 2$. Three types of curves are presented, each giving a mixing length as a function of travel distance. The solid line is the mixing length of the ensemble, as determined by our previous methodology. The various dotted lines are the mixing lengths of various single realizations. still computed as above, based on a least squares fit of the transverse spatial average of the concentrations in that realization to a complementary error function. Each dotted line represents a random variable. the single realization mixing length for the given geology and plume aspect ratio. Finally, the mean of this random variable, i.e. the expected value of the single realization mixing length, is shown in the dashed line.

From an operational point of view, the dashed line determines the rise time in concentration as observed with an infinitely sharply resolved instrument. The difference between the dashed line and the solid line is due to the uncertainty associated with predictions of plume arrival times. This uncertainty can be represented as the dispersion $\left\langle(R-\langle R\rangle)^{2}\right\rangle^{1 / 2}$ of the single realization mean position $R$ of the mixing zone. Note that $R$ is defined by transterse averages, but not ensemble averages, and is thus a random variable. It is represented in the plots by the crossed lines.

Depending on the specific question being studied, these two components of the ensemble 
based dispersion are each of importance. The fitting of recorded concentration data to parameters in simulation equations ior a well characterized and well instrumented site has more to do with the instrument rise time. but many other questions. such as the prediction of future concentration boundaries at an incompletely characterized site. have more to do with the variance of the single realization mean, considered as a random variable orer the ensemble, i.e. the concentration arrival time. The latter question (arrival times) is more commonly related to failures of hydrology predictions, and thus has an interest in its own right.

One consequence of our analysis is that the transient (i.e. preasymptotic) dispersirn is more important than the asymptotic dispersion, for heterogeneities which are active all length scales. See $[10,21,23,22]$

We have applied renormalized perturbation theory to the equation defining the dispersion tensor. At lowest order, this expansion is equivalent to Corrsin's hypothesis. A new derivation [23] of Corrsin's hypothesis shows its approximate validity for all times, and not just for long times, as is customarily assumed. This analysis has led to a determination of the asymptotically nonzero character of transverse dispersion, and improred upon earlier approximate solutions of Corrsin's hypothesis [17] Renormalized expansions (such as Corrsin 's hypothesis) are presumably more accurate at moderate amplitude of the expansion parameter (the fluctuating part of the velocity, $\delta \vec{v}$ ), but cannot be directly compared in accuracy to higher order primitive expansions, since each has terms missing in the other expansion. At small values of the expansion parameter, higher order primitive expansions should be more accurate than low order renormalized ones, but this is not the regime of practical interest.

The spreading of the mixing layer is characterized by the second moment $I^{-}$of the fluctuation $\vec{x}^{\prime}$ of the particle displacement $\vec{x}$. The equation for $\mathbb{X}$ is

$$
\frac{d^{2} \cdot X}{d t^{2}}=\frac{2}{(2 \pi)^{m}} \iint\left\langle\delta \vec{v}\left(k^{\prime} .0\right) \delta v^{*}(\vec{k} \cdot t) e^{i \vec{k} \cdot \vec{x}}\right\rangle d \vec{k} d \vec{k}^{\prime}
$$

where $m$ is the spatial dimension and the molecular diffusion coefficient is assumed to be zero. The triple product in the angle brackets introduces a closure problem: Corrsin's hypothesis is to declare the exponential independent of the velocities, leading to a closed equation. if $x$ is also assumed to be Gaussian. In [23] (see also [13]), a systematic expansion of the triple product integrand leads to a renormalized perturbation series. Moreorer. it is shown that under the assumptions that $x$ and $\delta \vec{v}$ are jointly Gaussian, and that $\delta \vec{r}$ is incompressible. all non-Corrsin terms vanish identically in this series. The basic idea is to expand the exponential, use Gaussian integral identities to evaluate monomial integrands in terms of the covariance. and resum the series. The result is an identity expressing the triple product integrand as

$$
\left\langle\delta \vec{\imath}\left(\vec{k}^{\prime} .0\right) \delta \vec{r}(\vec{k}, t)\right\rangle\left\langle e^{i \vec{k} \cdot \vec{x}}\right\rangle-\frac{1}{(2 \pi)^{m}} \int d t_{1} \int d t_{2} \int d \vec{k}_{1} \int d \vec{k}_{2} F
$$

where

$$
F=\left\langle\delta \vec{r}\left(\vec{k}^{\prime} \cdot 0\right) \delta \vec{v}\left(\vec{k}_{1}, t_{1}\right) e^{-i \vec{k}_{1} \cdot \vec{r}\left(t_{1}\right)}\right\rangle \cdot \vec{k} \times\left\langle\delta \vec{v}(\vec{k}, t) \delta v\left(\vec{k}_{2}, t_{2}\right) \epsilon^{-i \vec{k}_{2} \cdot \vec{r}\left(t_{2}\right)}\right\rangle \cdot\left\langle e^{i \vec{k} \cdot \vec{x}(t)}\right\rangle .
$$

This identity generates an iterated expansion. Assuming that $\vec{\imath}$ is incompressible. all completely expanded terms after the first vanish identically, which yields Corrsin is hyothesis. 
We have introduced a diagrammatic formulation of the renormalized perturbation theory equations to allow a simple conceptual understanding of the otherwise very complicated expressions which arise in higher order perturbation theory [23]. In Figure 4, we illustrate the graphical representation of the above equation. We use a solid black circle for the relocity

fluctuation $\delta \vec{v}$, a hollow circle for the phase factor due to the particle position $\epsilon^{-\vec{\imath} \cdot \vec{r}}$. and a horizontal straight line for the integrals over time and wave vector. These symbols are called the velocity vertex. position vertex, and integral line respectively. The quantities which are grouped together for the statistical average are linked by jagged lines called statistics lines. The vertical lines which are connected to an integral line give the dependence of the integrand of the integrals.

\subsection{Nonlinear Transport in Heterogeneous Media}

Both nonlinearity and heterogeneity cause dispersive mixing of fluid transport in porous media. Starting with the Buckley-Leverett equation as primitive transport equations. the nonlinear coupling to Darcy's law causes viscous fingering. The role of geological heterogeneities in velocity dispersion and channeling was discussed in §3.1. These two effects. however. tend to compete, rather than to be reinforcing. once the heterogeneities have served as a seed. to initiate the growth of nonlinear fingers. Thus we find that the relative strength of these two effects is important: as in $\S 3.1$, we believe that the intermediate time periods and transients in the dynamical history are also important.

In the simulations presented below, we have set gravity, capillary pressure. and molecular diffusion to zero, and we have used quadratic relative permeability functions. These equations are characterized by the oil to water viscosity ratio. We consider two values for this ratio, 4 and 10 . The first is marginally unstable to viscous fingering and the second is weakly. unstable. This data is then analyzed in two ways: dispersively, as in §3.1. and hyperbolically: as we now explain. The hyperbolic analysis starts from the same transversely averaged. ensemble averaged saturations, which depend on time and longitudinal distance. From this time dependent saturation. time dependent effective fractional flow functions. total mobility: and relative permeability functions are constructed. The motion of these reservoir functions in time is the renormalization group (RNG) dynamics for the equations themselves. See also $[16,12]$

In Figure 5, we show three levels of nonlinearity, for each of three levels of heterogeneity: with finite length scale correlated $(\beta=\infty)$ geology. The trend as shown in the three frames is that more strongly nonlinear flow displays the most dispersion. followed by the weakly nonlinear flow, and finally the linear flow. But the degree to which these statements hold is much stronger in the case of weak geological heterogeneities. Figure 6 displays the same trends, in the case of multiple length scale heterogeneities $(\beta=0.5)$.

In Figures 7 and 8 , we show a hyperbolic (RNG) analysis for the time dependent fractional flow function. The basis of this analysis is as follows. We define an ensemble and transierse averaged fractional saturation $\tilde{s}(x, t)$, where $x$ denotes the longitudinal coordinate. parallel to the average flow. This function can be inverted to define $x(\dot{s}, t)$. Lising $\dot{s}(x, t)$, we determine a renormalized $\tilde{f}^{\prime}$ by numerical differentiations as

$$
\tilde{f}^{\prime}=\frac{\langle x(\tilde{s} \cdot t+\Delta t)-x(\tilde{s} \cdot t)\rangle}{\left.\Delta t<v^{\prime}\right\rangle}
$$


and then find $\dot{f}$ by integration of $\dot{f}$. Figure $\vec{i}$, with the finite length scale correlations $(3=\infty)$ for its geology: and mixing length exponents significantly smaller than one. shows the expected convexified shape as the R.TG fixed point. Figure 8, with multi-length scale correlated geology $(3=0.5)$, has mixing length scaling exponents closer to one. but still shows no influence from renormalization. i.e. it equals the convex envelope of the primitive fractional flow function. The lack of significant renormalization in the correlated case requires further study. but indicates that for the parameters considered here. dispersion renormalization is more appropriate than hyperbolic renormalization.

\section{References}

[1] E.E. Adams and L. Gelhar, Field Study of Dispersion in a Heterogeneous Acquifer ?. Spatinl . Woment Analysis, Water Resources Research, 28(1992). pp. 3293-330т.

[2] M. Avellaneda and A. Majda. Mathematical .Models with Exact Renormalization for Turbulent Transport. Comm. Math. Phys.. 131(1990), pp. 381-t?29.

[3] J. Cannon, Continuous Sample Paths in Quantum Field Theory. Comm. Math. Phy̌s.. $35(1974)$, pp. $215-234$.

[4] P. Colella and O. Lanford. in Constructive Quantum Field Theory. G. Velo and A. Wightman. eds.. Springer-Verlag. New York, 1973.

[5] G. Dagan, Dispersion of a Passive Solute in Non-ergodic Transport by Steady l'flocity Fields in Heterogencous Formations. J. Fluid Mech, 233(1991). pp. 1281-1290.

[6] The Significance of Heterogeneity of Evolving Scales to Transport in Porous Formations. Water Resources Research, 30(1994), pp. 3:327-3:3:36.

[i] F. Furtado. J. Glimm. W.B. Lindquist and F. Pereira, Characterization of . Mirmng Length Growth for Flow in Heterogeneous Porous Media, SPE 2123:3, 1991.

[8] L.W. Gelhar, A. Mantoglou, C. Welty and K.R. Rehfeldt. A Revieu of Field-Scale Physical Solute Transport Processes in Saturated and Lnsaturated Porous . Media. EPRI Report EA-4190. 198.5.

[9] J. Glimm and A. Jaffe. Quantum Physics: A Functional Intergral Point of lieu. 2nd edition, Springer-Verlag, Vew York. 1987.

[10] J. Glimm. W.B. Lindquist. F. Pereira and Q. Zhang, A Theory of . Macrodrspersion for the Scale L"p Problem. Transport in Porous Media. 13(1993). pp. 97-122.

[11] I.A. Ibragimor and Y.A. Rosanor, Gaussian Random Processes (English Translation:. Springer-Verlag. . Jew York. 1978.

[12] P.R. King, A.H. Muggeridge and W.G. Price, Renormalization C'alculations of Immiscible Flou. Transport in Porous Media. 12(1993). pp. 237-260. 
[13] R.H. Kraichnan. Lagrangian Velocity Covariance in Helical Turbulence. J. Fluid Mech.. 81(19Ti). pp. 38.5-398.

[14] M.I. Morris and R.C. Ball, Renormalization of . Viscible Flow in Hierarchically Correlated Heterogeneous .Media. Cavendish Laboratory Preprint.

[15] Renormalization of Miscible Flow in Uncorrelated Heterogeneous Media. Cavendish Laboratory Preprint.

[16] _ Renormalization of Miscible Flow Functions, J. Phys. A. 23(1990). pp. 41994209.

[17] S. Neuman and Y.-K. Zhang, A Quasi-Linear Theory of Non-Fickian and Fickian Subsurface Dispersion I. Theoretical Analysis with Application to Isotropic Media. Water Resources Research, 26(1990), pp. 887-902.

[18] H. Rajaram and L.W. Gelhar. Plume Scale-dependent Dispersion in Heterogeneous Aquifers, 2. Eulerian Analysis and Three Dimensional Aquifers. Water Resources Research, 29(1993), pp. 3261-32 11.

[19] E.A. Sudicky, A Natural Gradient Experiment on Solute Transport in a Sand Aquifer: Spatial Variability of the Hydraulic Conductivity and Its Role in the Dispersion Process. Water Resources Research. 22(1986), pp. 2069-2082.

[20] T. Wallstrom. Turbulent Diffusion Phase Transition is Due to Viscosity-Dependent Energy Spectrum, LANL Report LA-UR-95-843, 1995.

[21] Q. Zhang, A :Multi-Length Scale Theory of the Anomalous : Yixing Length Grouth for Tracer Flow in Heterogeneous Porous Media, J. Stat. Phys.. 66(1991), pp. 48.5-.501

[22] _. The Asymptotic Scaling Behavior of Mixing Induced by a Random lelocity Field, Adv. Appl. Math., 16(1995), 23-58.

[23] _ The Transient Behavior of Mixing Induced by a Random I'elocity Field. Water Research Resources, 31(1995), pp. 57i-591.

[24] Q. Zhang and J. Glimm, Inertial Range Scaling of Laminar Shear Flow as a Model of Turbulent Transport. Comm. Math. Phys.. 146(1992), pp. 21i-229. 


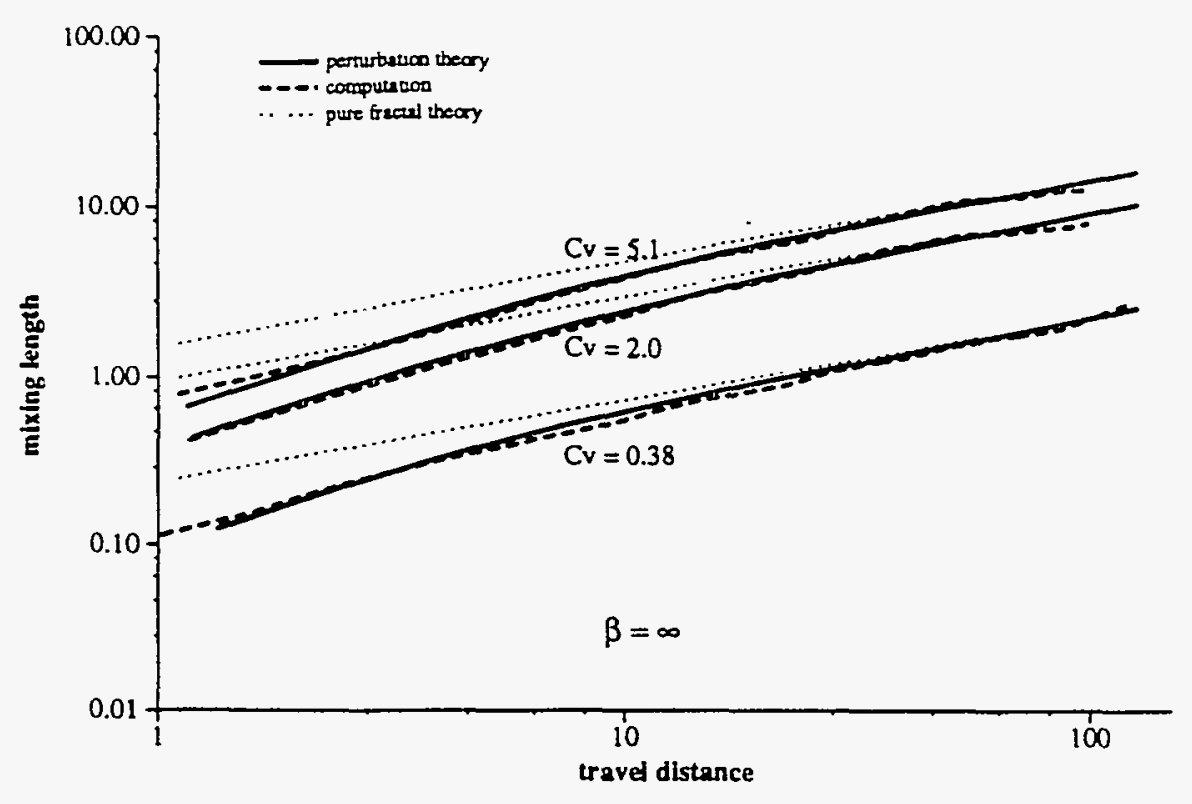

Figure 1: Log-log plot of mixing length, $l$, as a function of travel distance for $3_{x}=x$. Each triple of curves shows a comparison between pure fractal theory. second order transport perturbation theory including transients, and numerical simulations. Each triple of curves corresponds to a different value of the coefficient of variation $C_{v}$ of the permeability field. as labeled.

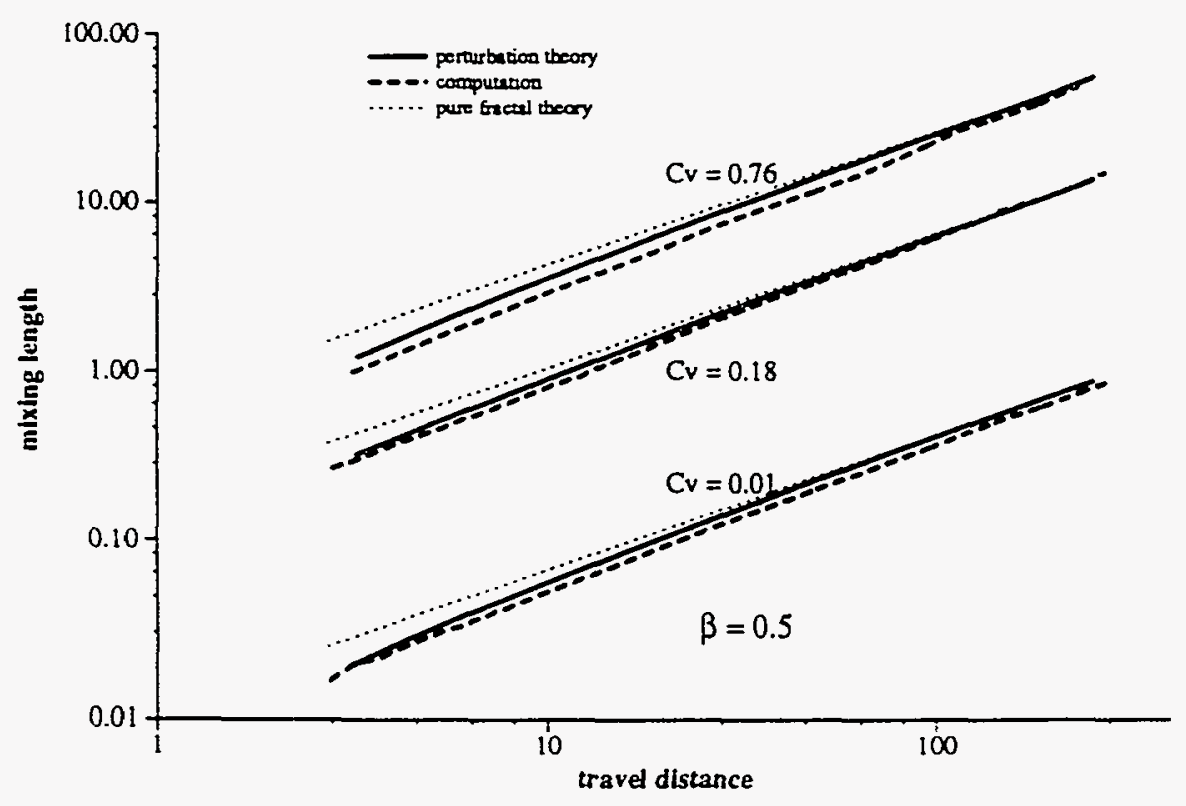

Figure 2: $\log \log$ plot of mixing length. $l$, as a function of travel distance for $3_{x}=0.5$. Each triple of curves shows a comparison between pure fractal theory. second order transport perturbation theory including transients, and numerical simulations. Each triple of curves corresponds to a different value of the coefficient of variation $C_{v}$ of the permeability field. as labeled. 


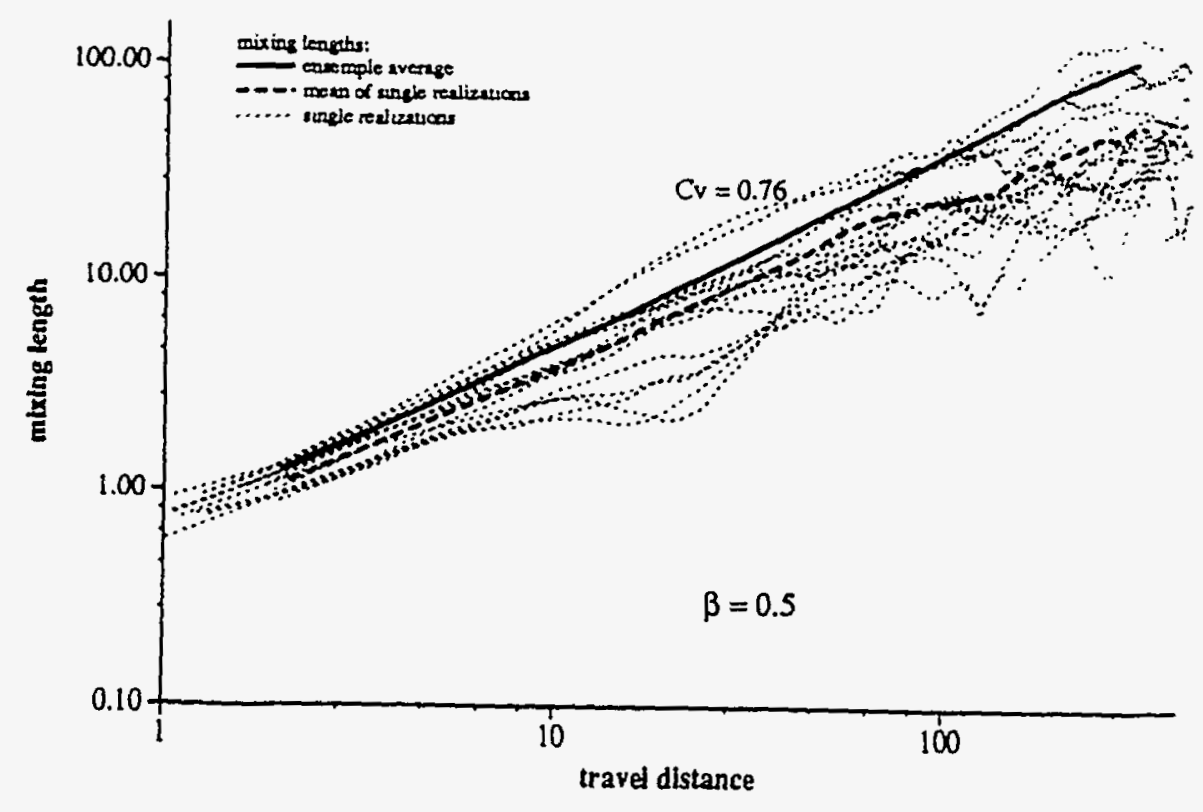

Figure 3: (a) Comparison of the ensemble mixing length (solid curve), the ensemble of single realization mixing lengths (various dotted curves), and the mean of the single realization mixing lengths (dashed curve), for a narrow channel (1:2 aspect ratio).

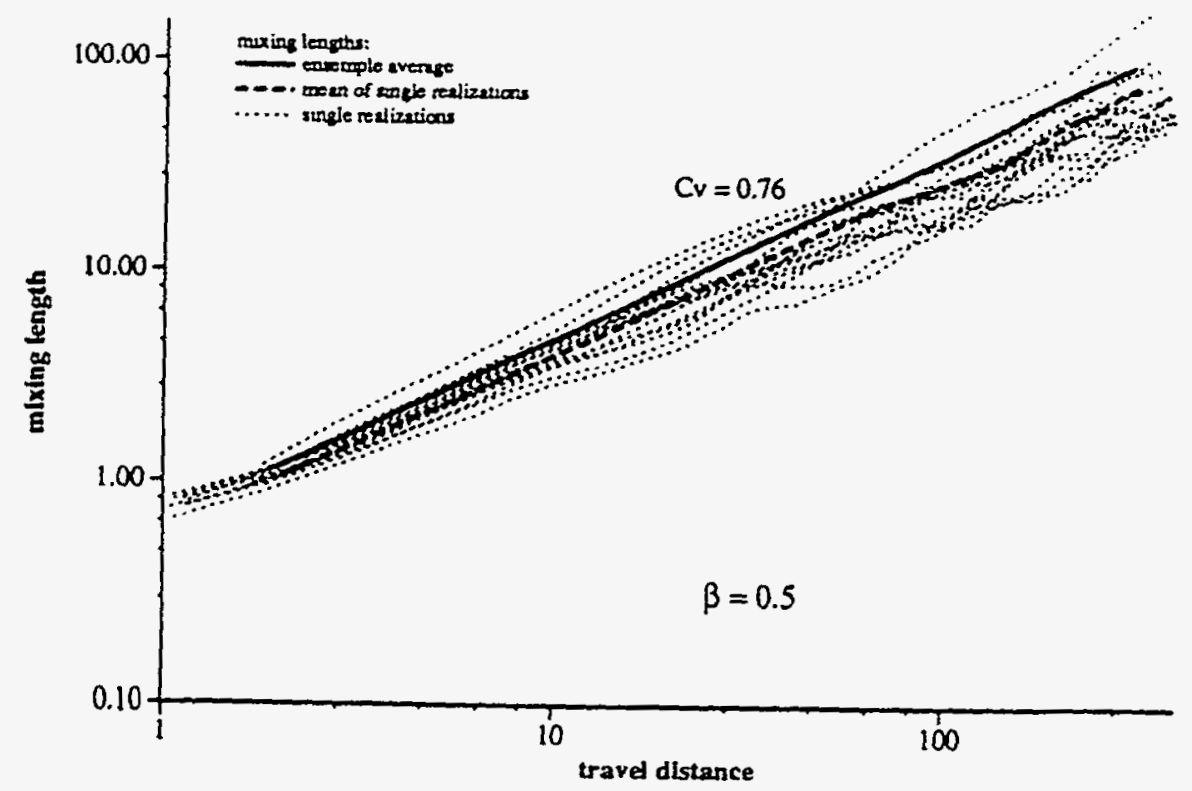

Figure 3: (b) Comparison of mixing lengths, as in Figure 3(a), but for a wide channel $11: 1$ aspect ration). 


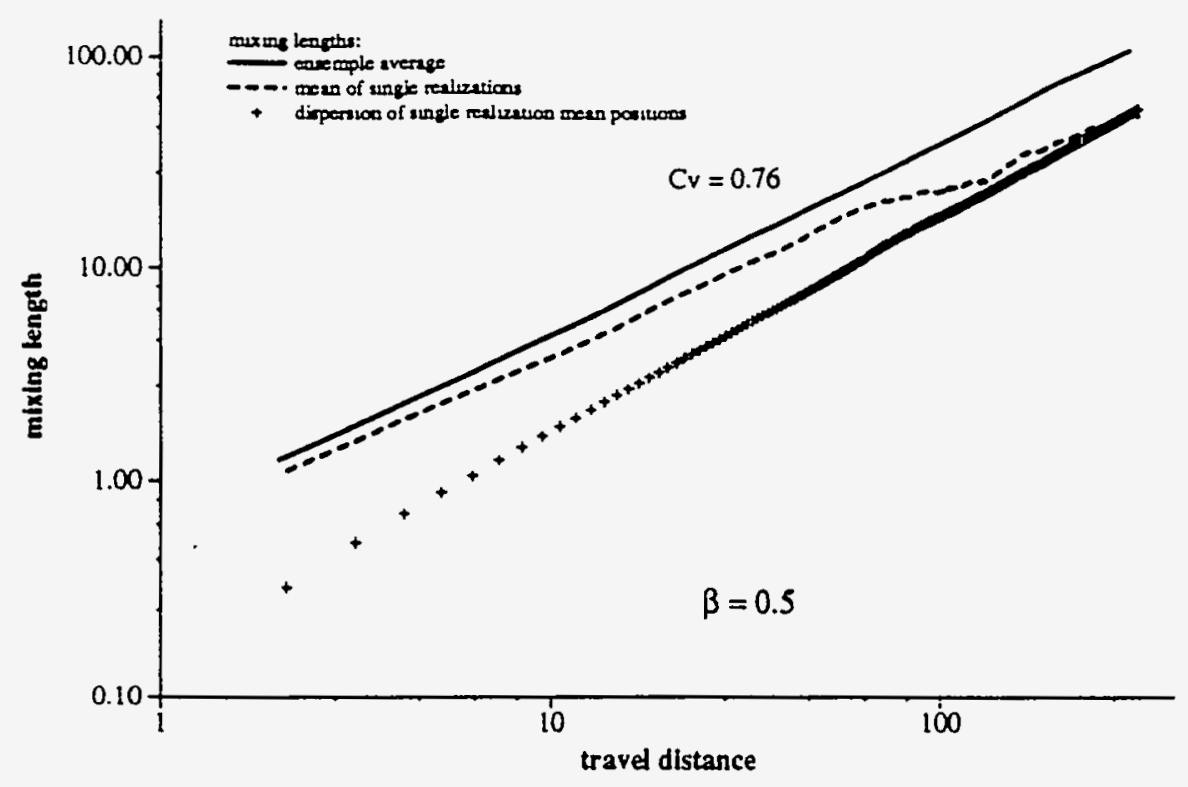

Figure 3: (c) Comparison of the ensemble mixing length (solid curve), the mean of the single realization mixing lengths (dashed curve), and apparent mixing length due to the dispersion of the single realization mean positions (crossed curve), for the narrow channel (1:2 aspect ratio).

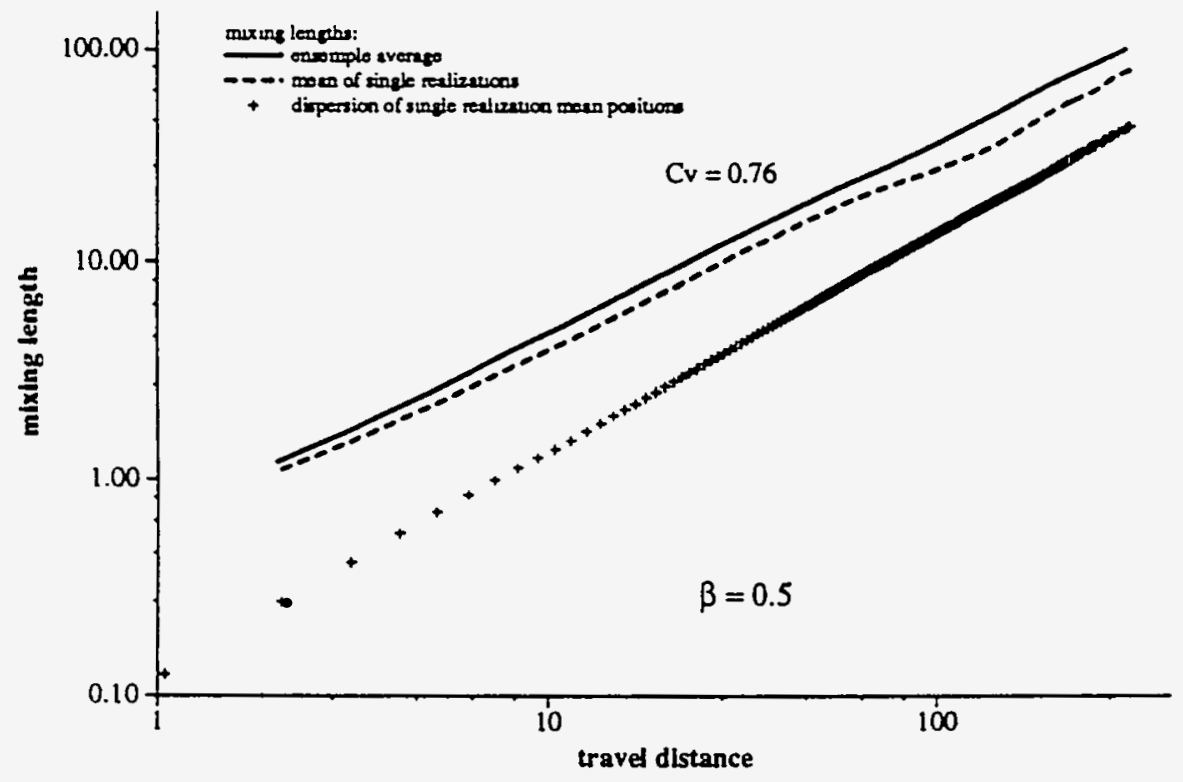

Figure 3: (d) As in Figure 3(c), but for a wide channel (1:1 aspect ratio). 


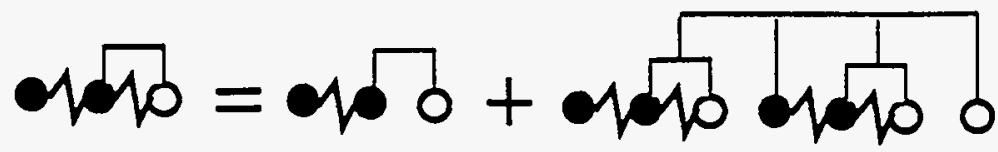

Figure 4: A diagrammatic representation of the mathematical equation for the dispersion tensor. The identity of the left hand side with the first subgraph on the right hand side (i.t. the vanishing of the second subgraph on the right hand side) represents Corrsin's hypothesis.

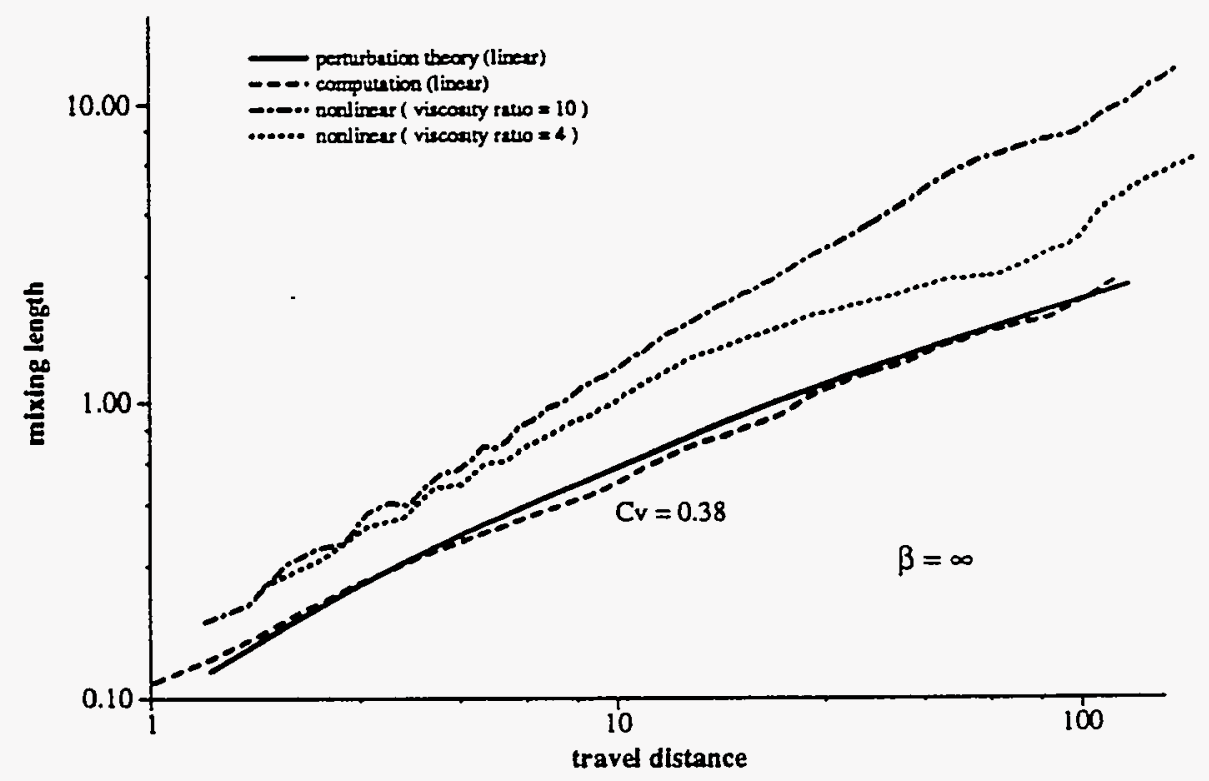

Figure 5: (a) Comparison of mixing length vs. travel distance for a low heterogeneity $\left(C_{v}=.38\right)$, uncorrelated $(\beta=\infty)$ ensemble of geologies. The solid line is a perturbation theory for the case of linear transport, while the dashed line is the computational result for the same case. The dotted and dot-dashed lines represent nonlinear (Buckley-Lererett) transport, with viscosity ratios of 4 and 10 respectively. 


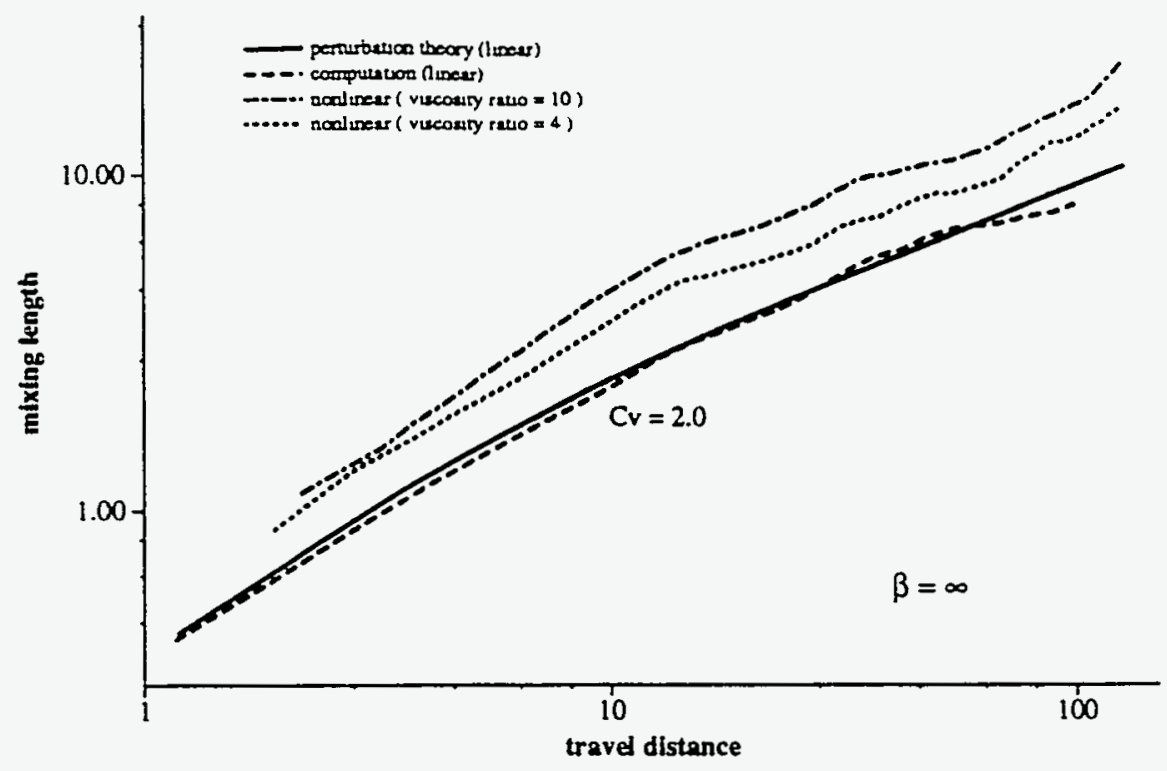

Figure 5: (b) . Mixing length is. travel distance as in Figure 5(a), for uncorrelated geological heterogeneities of moderate strength coefficient of variation, $C_{v}=2.0$.

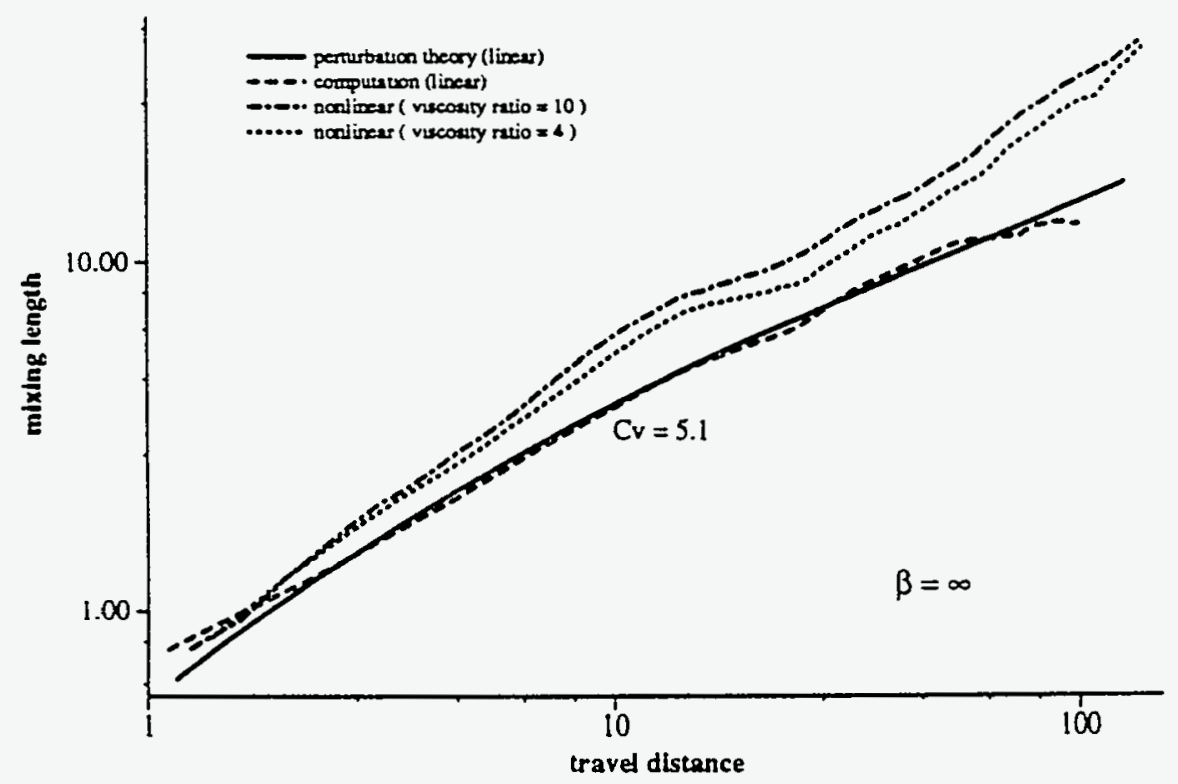

Figure 5: (c) As in Figure $5(\mathrm{a})$. $\mathrm{j}(\mathrm{b})$. for uncorrelated geological heterogeneities of high strength coefficient of rariation. $C_{v}=5.1$. 


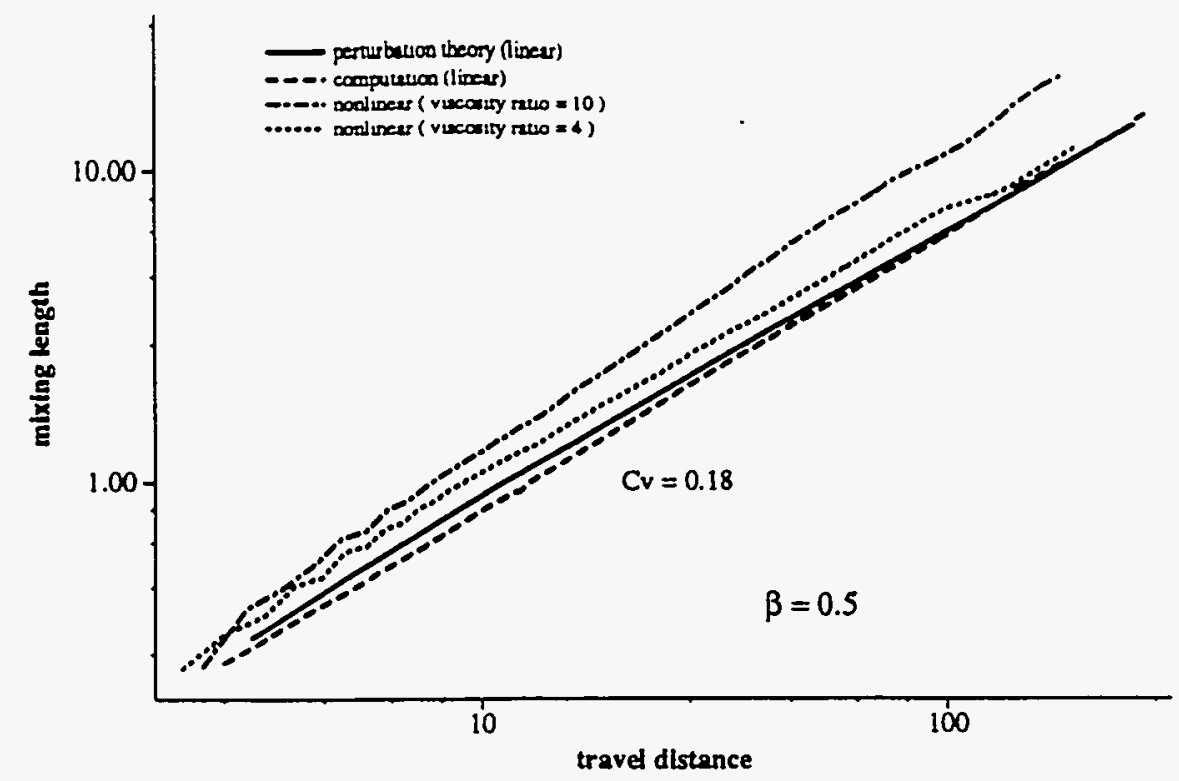

Figure 6: (a) The mixing length for a fractally correlated ensemble of geologies. of low strength coefficient of variation, $C_{v}=.18$.

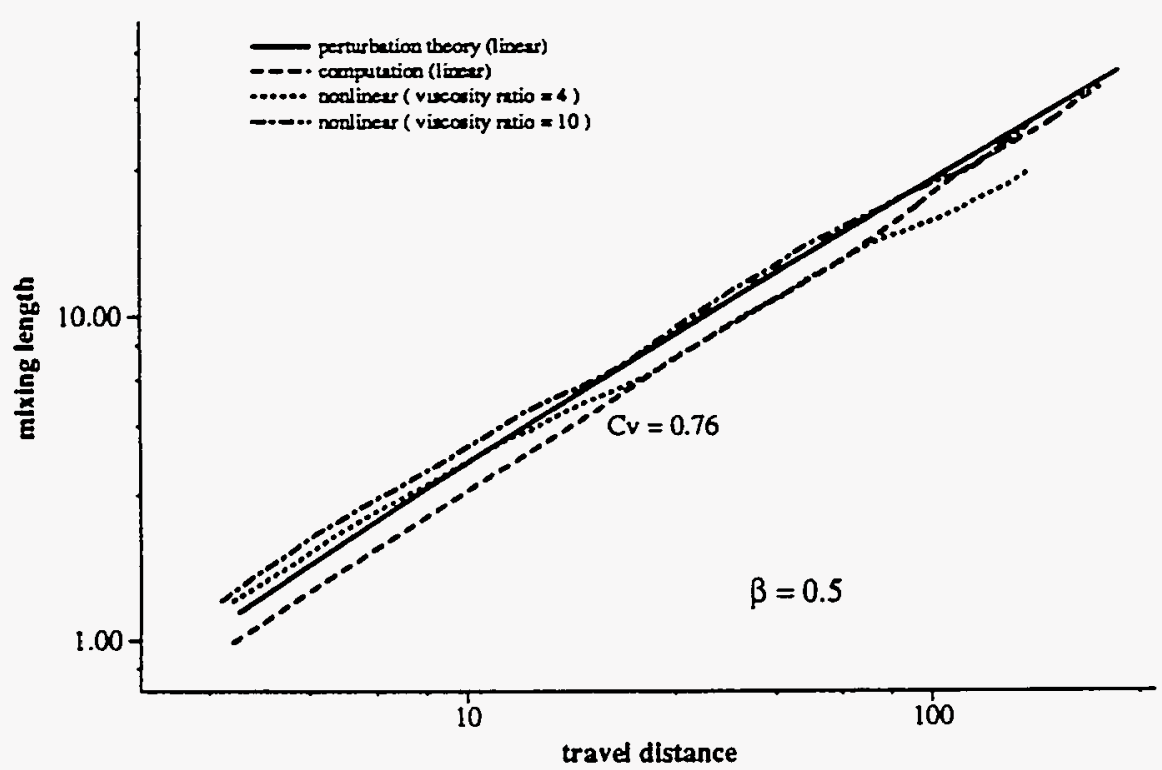

Figure 6: (b) The mixing length for a fractally correlated ensemble of geologies. of moderately high strength coefficient of variation, $C_{v}=.76$. 


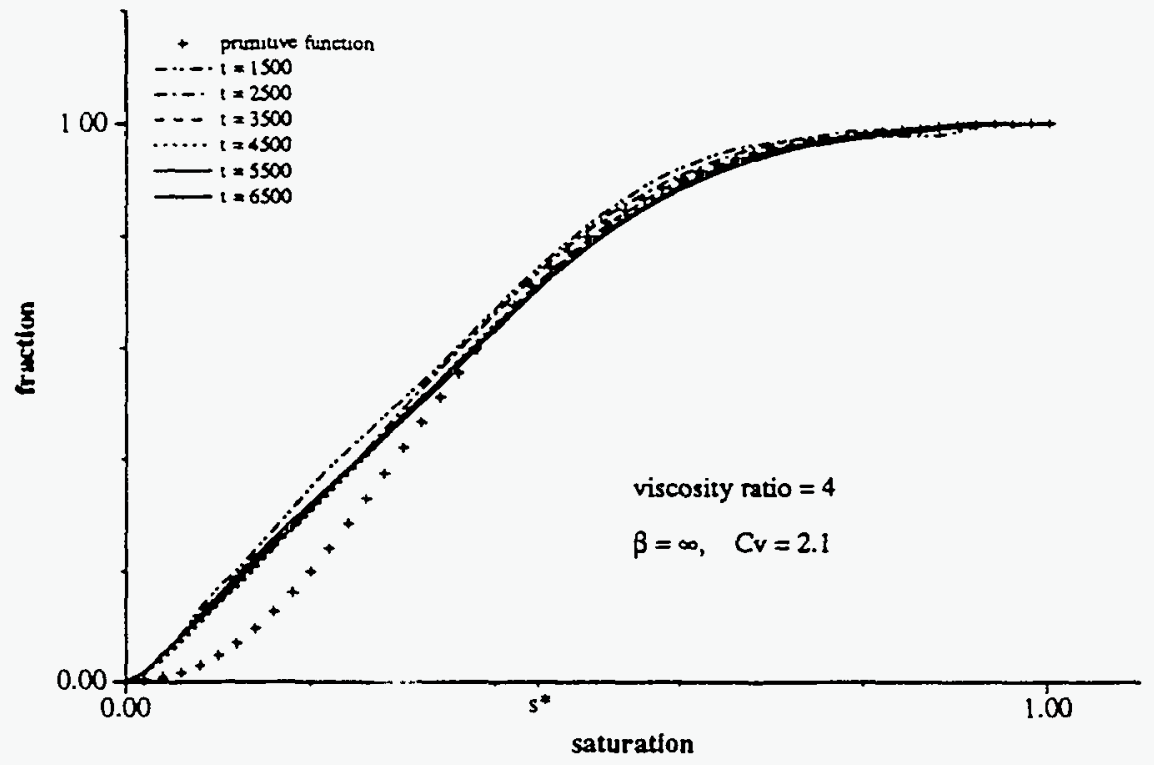

Figure $i$ : Hyperbolic renormalization of the fractional flow function $f$ for the case of uncorrelated heterogeneities. The figure shows a plot of $\tilde{f}(\tilde{s})$ for a sequence of times. with a well defined $t \rightarrow \infty$ limit (a RNG fixed point). The primitive fractional flow function $f(s)$ is plotted for comparison. as a crossed curve.

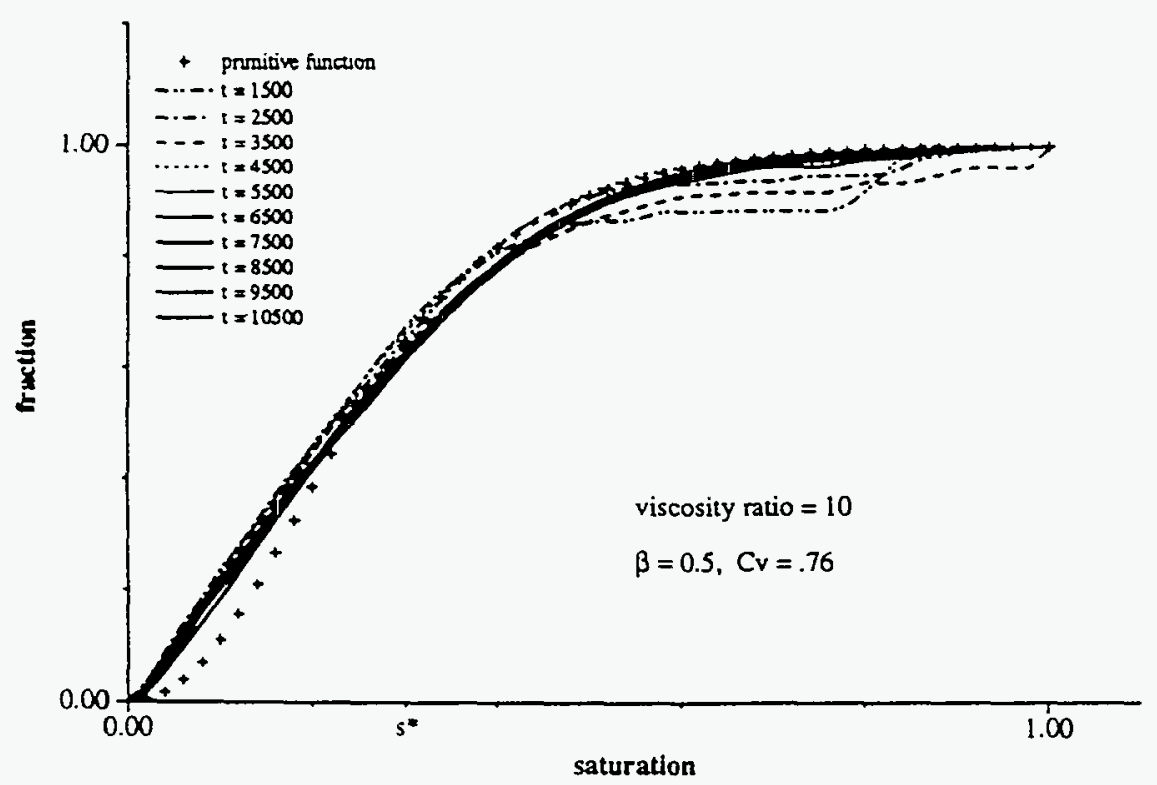

Figure 8: Hyperbolic renormalization of the fractional flow function $f$ for the case of correlated (fractal) heterogeneities. The primitive fractional flow function $f(s)$ is plotted as a crossed curve. for comparison. The absence of significant renormalization indicates that dispersive, rather than hyperbolic renormalization will be more appropriate for the fluid and geological parameters considered here. 